\title{
Evaluación oftalmológica en niños derivados a un proyecto de rehabilitación en baja visión de un organismo de apoyo social
}

\author{
Ophthalmological evaluation in children referred to a low-vision rehabilitation \\ project of a social assistance agency
}

\author{
Barría Von-B. Fernando ${ }^{\mathrm{a}, \mathrm{b}}$, Parada H. Rodrigo ${ }^{\mathrm{a}}$, Triviño F. Loreto ${ }^{c}$, \\ Ramos G. Patricia ${ }^{\mathrm{d}}$, Marín D. Marta ${ }^{\mathrm{e}}$
}

\author{
aservicio de Oftalmología Hospital Guillermo Gran Benavente de Concepción \\ ${ }^{b}$ Comité prevención de ceguera de la Asociación Panamericana de Oftalmología \\ 'Residente de Oftalmología, Facultad de Medicina, Universidad de Concepción \\ 'Terapeuta Visual, Equipo de Baja Visión, Facultad de Medicina, Universidad de Concepción. Asesora en Baja Visión para América Latina \\ de CBM-ICEVI \\ eTecnólogo Medico, especialista en oftalmología, Equipo de Baja Visión, Facultad de Medicina, Universidad de Concepción
}

Recibido: 29 de enero de 2018; Aceptado: 27 de diciembre de 2018

\section{Resumen}

Un niño con baja visión (BV) rehabilitado puede tener una educación integral. Objetivo: Estudiar el perfil de los escolares derivados para evaluación a un Proyecto de Rehabilitación en un organismo de apoyo social. Pacientes y Método: Estudio transversal descriptivo de beneficiarios evaluados entre septiembre 2015 y septiembre 2016 en JUNAEB. Se consideró diagnóstico de derivación, agudeza visual monocular (AV) con corrección óptica para lejos (cartillas Feinbloom) y cerca (cartillas Zeiss). Se clasificaron según $A V$ y perimetría. Se consideró éxito de tratamiento lograr $A V \geq 0,4$ para lejos y/o cerca con ayuda óptica. Resultados: Se evaluaron 278 escolares. 153 (55\%) fueron hombres, $121(43,5 \%)$ entre 10 a 14 años. Catarata congénita bilateral, distrofias retinales, alta miopía, atrofia óptica y nistagmus congénito fueron las patologías más frecuentes. $224(80,6 \%)$ se habilitaron con ayudas. $85(37,9 \%)$ presentaron BV moderada y $63(28,6 \%)$ BV severa. $122(54,5 \%)$ presentaban perimetría normal, $68(30,4 \%)$ campo visual $(\mathrm{CV})$ tubular, $19(8,5 \%)$ defecto sectorial del CV y $15(6,7 \%)$ compromiso central del CV. $198(88,4 \%)$ escolares lograron éxito visual de lejos y todos lograron éxito visual de cerca. 48 (17,2\%) escolares no pudieron ser rehabilitados, estando asociados a una afección neuroftalmológica $(41,7 \%)$, alto error refractivo $(16,6 \%)$ o glaucoma congénito $(10,4 \%) .6(2,2 \%)$ casos mejoraron su AV con nueva corrección óptica. Conclusión: El éxito obtenido demuestra la necesidad de habilitar visualmente a escolares con BV. Nuestro desafío es mantener este programa y educar al oftalmólogo prestador para una derivación oportuna.
Palabras clave:

Niños;

baja visión;

ayudas baja visión;

baja visión/

rehabilitación

Correspondencia:

Rodrigo Parada $\mathrm{H}$.

rodrigoparada.h@gmail.com 


\section{Abstract}

Introduction: A low-vision (LV) rehabilitated child can receive comprehensive education. Objective: To study the profile of school children referred for evaluation to a rehabilitation project in a social assistance agency. Patients and Method: Descriptive cross-sectional study of beneficiaries evaluated between September 2015 and September 2016 in the National Board of School Assistance and Scholarships (JUNAEB). The referral diagnosis, monocular visual acuity (VA) with optical correction at far (Feinbloom chart) and close (Zeiss chart) distances were considered. They were classified according to VA and perimetry. Treatment success was considered if VA reaches $\geq 0.4$ at far and/or close distances with optical devices. Results: 278 students were assessed. 153 (55\%) were men, 121 (43.5\%) between the ages of 10 to 14 . Bilateral congenital cataract, retinal dystrophies, high myopia, optic atrophy, and congenital nystagmus were the most frequent pathologies. 224 students (80.6\%) received optical devices. 85 (37.9\%) presented moderate LV and 63 (28.6\%) severe LV; 122 (54.5\%) presented normal perimetry, 68 (30.4\%) tubular Visual Field (VF), 19 (8.5\%) sectoral VF defects, and $15(6.7 \%)$ central scotoma. 198 (88.4\%) students achieved visual success at a far distance and all achieved visual success at a near distance. 48 (17.2\%) students could not be rehabilitated due to a neuro-ophthalmological condition $(41.7 \%)$, high refractive error $(16.6 \%)$ or congenital glaucoma (10.4\%).Six (2.2\%) cases improved VA with a new optical correction. Conclusion: This success demonstrates the need to provide low vision aids to schoolchildren with LV. Our challenge is to maintain this program and to educate ophthalmologist for timely referral.

\section{Keywords:}

Children;

low vision;

low vision aids;

low vision/

rehabilitation

\section{Introducción}

Según la Organización Mundial de la Salud (OMS), "una persona con baja visión (BV) presenta disminución de su función visual con agudeza visual (AV) menor a $0,3(20 / 60,0,6 \log \mathrm{MAR})$ y/o campo visual (CV) menor a $20^{\circ}$ desde el punto de fijación, en su mejor ojo, no susceptible de mejorar con corrección óptica convencional ni con tratamiento médico y/o quirúrgico, pero que podría usar su visión remanente para planificar o ejecutar tareas especificas" ${ }^{\prime \prime 2}$. Por otro lado una "persona ciega" tiene una visión menor a 0,05 (3/60, $1,3 \log$ MAR) y/o un campo menor de $10^{\circ}$ en el mejor ojo con la mejor corrección óptica posible. La OMS estima que 19 millones de niños en el mundo presentan discapacidad visual y de ellos 1,4 millones son ciegos y 17,6 tienen un remanente de visión ${ }^{1}$. Al año 2007 en Chile se estimaba que 90.000 adultos $^{3}$ podrían rehabilitarse visualmente, pero no son derivados por desconocimiento y/o falta de servicios. No existen estimaciones nacionales de niños afectados. La discapacidad visual en niños afecta su desarrollo psicológico, educativo y socioeconómico, generando una carga de costos a la comunidad y aunque su prevalencia es baja, cobra importancia por su larga sobrevida. Las estrategias en prevención de ceguera actuales han permitido que muchos casos no terminen ciegos, sino con una deficiencia visual que tiene profundas implicancias funcionales y psicológicas en la infancia y que a futuro limitará su desempeño educacional y/o laboral ${ }^{4}$. Esta situación puede revertirse con una rehabilitación visual, donde mediante entrenamiento y habilitación en el uso de dispositivos ópticos, no ópticos y/o electrónicos, los afectados aprenden a maximizar su remanente visual, permitiéndoles integrarse a su comunidad ${ }^{5}$. La etiología de la discapacidad visual infantil varía en las diferentes regiones del país, requiriéndose información específica para adaptarse a las necesidades locales y erradicar las causas prevenibles. No existen estadísticas completas que permitan estimar la prevalencia de BV en escolares chilenos ni evaluación de las causas. Un análisis clínico de 46 niños con BV ingresados a la Corporación de ayuda al limitado visual "COALIVI", centro especializado de Concepción, comunicó que sus principales causas de discapacidad visual fueron malformaciones oculares, seguidas de catarata congénita bilateral y retinopatía del prematuro $(\mathrm{ROP})^{3}$. Un articulo del año 2000, muestra la prevalencia de una deficiencia visual asociada a error refractivo en la población infantil en Florida era del 7\% y que 50 niños $(0,95 \%)$ presentaban una visión menor o igual a $0,25^{6}$. Otro estudio mas reciente, referido a prevalencia de error refractivo en escolares, se detectaron dos niños $(0,04 \%)$ con baja visión integrados en colegio regular, que usaban ayudas ópticas proporcionadas por JUNAEB, indicando su inclusión al sistema educativo ${ }^{6,7}$.

Nuestro objetivo es mostrar la experiencia nacional en atención de escolares con $\mathrm{BV}$, derivados al proyecto de rehabilitación de la Junta nacional de Auxilio escolar y becas (JUNAEB), caracterizando la población escolar afectada en Chile, sus etiologías y resultado de su rehabilitación visual. Consideramos que esta información permitirá educar al oftalmólogo y pediatra para una derivación precoz, facilitará el diseño de medidas 
preventivas de patologías causantes de BV infantil y la planificación de servicios de rehabilitación visual necesarios para la detección, tratamiento y vigilancia de estudiantes en esta condición.

\section{Pacientes y Método}

Se realizó un estudio transversal descriptivo de escolares provenientes del sistema educacional publico de Chile que son detectados en consulta refractiva por prestadores de JUNAEB a nivel nacional, siendo derivados al proyecto de rehabilitación en BV de JUNAEB, para ser evaluados por el equipo de BV de la Facultad de Medicina Universidad de Concepción, bajo modalidad de licitación publica, realizando operativos en las regiones entre septiembre de 2015 a septiembre de 2016. Los niños se caracterizaron según edad, sexo, diagnóstico de derivación al proyecto, mejor agudeza visual (AV) monocular corregida para distancias de lejos y cerca y perimetría. Se realizó examen de AV monocular de lejos, con optotipos numéricos de Feinbloom (Desings for Vision inc.) a distancia de 3 metros (figura 1) y para cerca con test de visión próxima de Zeiss, montado en atril de lectura, a distancia de 25 centímetros. En casos iletrados se utilizó test de LEA Hivarinen para lejos y cerca (figura 1). Se consideró la mejor AV alcanzada en su mejor ojo con corrección óptica. Se realizó estudio de perimetría monocular me- diante pantalla tangente de Bjerrum a distancia de 1 metro. Se consideró la mejor perimetría lograda con corrección óptica.

Los pacientes se clasificaron según criterios de visión y de perimetría. Considerando la AV de lejos, en el mejor ojo con la mejor corrección óptica se utilizó la clasificación estadística internacional de enfermedades y problemas de salud, $10^{\text {a }}$ revisión $(\mathrm{ICD}-10)^{8}$ de la Organización mundial de la salud en su versión del 2016, siendo similar a la norma de severidad de BV propuesta por Faye ${ }^{9}$ y a la clasificación de la deficiencia visual (9D90) de la versión IC-11 de la OMS. Se clasifican en baja visión moderada, BV severa y BV Profunda (tabla 1), considerando esta última como ceguera funcional y legal con remanente visual y la OMS considera una categoría adicional para discapacidad visual no calificada.

Según mejor perimetría, se clasificaron de acuerdo con los defectos de CV dominantes en 4 grupos funcionales $^{9}$ (GF), considerando: GF1 presenta compromiso central del CV, GF2 presenta compromiso periférico del CV, GF3 tiene defectos sectoriales del CV y GF4 no presenta alteraciones del CV. Se prescribieron ayudas ópticas y no ópticas, para cada paciente, para distancia de $3 \mathrm{~m}$ (lejos) y distancia de lectura a $25 \mathrm{~cm}$ (cerca), realizando entrenamiento y supervisión para su correcto uso.

Se consideró éxito de tratamiento de acuerdo con el protocolo de baja visión de la Fundación ONCE para la

Tabla 1. Categoría de Baja Visión según clasificación IC-10

\begin{tabular}{|c|c|c|}
\hline Categoría deficiencia visual* & Peor que**: & Igual o mejor que: \\
\hline Baja Visión Moderada & $20 / 60 \quad(6 / 18-0,3 \quad 0.6 \log M A R)$ & $(6 / 48-0,125$ 0,9LogMAR) \\
\hline Baja Visión Severa & 20/200 (6/60 - 0,1 1.OlogMAR) & (6/120 - 0,05 1,3logMAR) \\
\hline Baja Visión Profunda & $20 / 500(6 / 150-0,04)$ & $20 / 1.000(6 / 300-0,02)$ \\
\hline
\end{tabular}

*Clasificación OMS ICD-10 Version: 2016. **Escala de vision: US feet (UK metrics / decimal / logMAR).

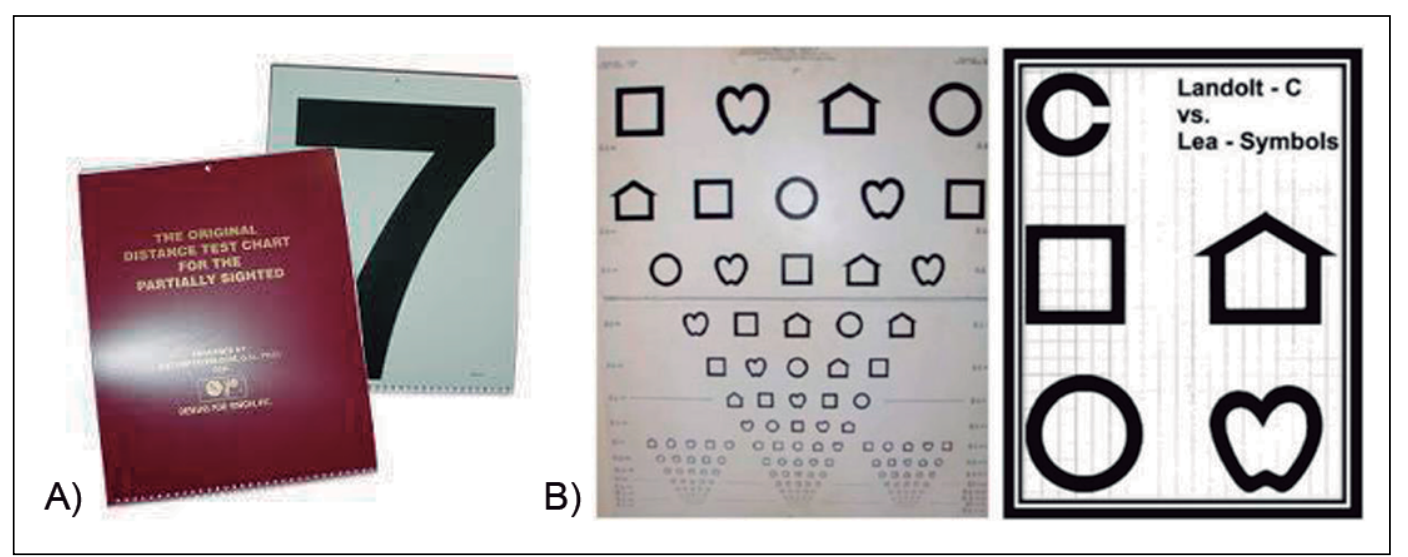

Figura 1. Cartillas de optotipos numéricos de Feinbloom (A) y test de LEA Hivarinen (B). Fotos de archivo personal de Sra. Patricia Ramos. 
cooperación e inclusión social de personas con discapacidad de España ${ }^{10}$, que considera como éxito si lograron AV para lejos mayor o igual a 0,4 con ayuda óptica, pues con dicha visión se alcanza un rango funcional que permite visión de pizarrón a 3 metros. Se consideró éxito de tratamiento para cerca, si lograron una $\mathrm{AV}$ mayor o igual a 0,4 con ayuda técnica, pues con dicha visión se alcanza un rango funcional que permite lectura de textos escolares con optotipos estándar ${ }^{10}$. El objetivo del programa de rehabilitación visual, es entrenar el resto visual y dotarle de los instrumentos ópticos y no ópticos que le ayuden a realizar las acciones cotidianas de cerca y de lejos que necesite. Todo el proceso de rehabilitación visual contempla la evaluación de las necesidades de cada persona, el diagnóstico y pronóstico de su patología, la evaluación funcional de la visión, el entrenamiento en el uso del resto visual y de las ayudas ópticas seleccionadas.

Los diagnósticos de derivación fueron definidos por el médico oftalmólogo tratante de cada escolar y extraídos de su interconsulta. En casos con más de un diagnóstico, se consideró el que originara mayor deterioro visual y/o constituyera una condición prevenible. Los datos fueron tabulados con Microsoft Excel ${ }^{\mathrm{MR}}$ para su análisis.

\begin{tabular}{|c|c|c|c|}
\hline Edad (años) & $\begin{array}{l}\text { Masculino } \\
\text { Número (\%) }\end{array}$ & $\begin{array}{l}\text { Femenino } \\
\text { Número (\%) }\end{array}$ & $\begin{array}{c}\text { Total } \\
\text { Número (\%) }\end{array}$ \\
\hline Menor 5 años & $3 \quad(2,0)$ & $1 \quad(0,8)$ & $4 \quad(1,4)$ \\
\hline 5 a 9 años & $49 \quad(32,0)$ & $44 \quad(35,2)$ & $93 \quad(33,5)$ \\
\hline 10 a 14 años & $74 \quad(48,4)$ & $47 \quad(37,6)$ & $121 \quad(43,5)$ \\
\hline Igual o mayor 15 & $27 \quad(17,6)$ & $33(26,4)$ & $60 \quad(21,6)$ \\
\hline Total & $153(100,0)$ & $125(100,0)$ & $278(100,0)$ \\
\hline
\end{tabular}

\begin{tabular}{|c|c|c|c|}
\hline Baja visión* & $\begin{array}{l}\text { Habilitado } \\
\text { Número (\%) }\end{array}$ & $\begin{array}{c}\text { No habilitado } \\
\text { Número (\%) }\end{array}$ & $\begin{array}{l}\text { Total } \\
\text { Número }\end{array}$ \\
\hline Moderada & $76 \quad(33,9)$ & $14(29,2)$ & $89 \quad(33,1)$ \\
\hline Severa & $85 \quad(38,0)$ & $1 \quad(2,1)$ & $86 \quad(31,6)$ \\
\hline Profunda & $63 \quad(28,1)$ & $4 \quad(8,3)$ & $67 \quad(24,6)$ \\
\hline Sin fijación & $0 \quad(0)$ & $25 \quad(52,1)$ & $25 \quad(9,2)$ \\
\hline No coopera & $0 \quad(0)$ & $4 \quad(8,3)$ & $(1,5)$ \\
\hline Total & $224(100,0)$ & $48(100,0)$ & $272(100,0)$ \\
\hline
\end{tabular}

*Clasificación OMS ICD-10 Version: 2016.

\section{Resultados}

Se obtuvieron registros de 278 escolares provenientes de todo Chile, que corresponden a ingresos de varios años que son evaluados en operativos. De ellos, $153(55 \%)$ casos eran de sexo masculino y 125 (45\%) femenino, distribuidos en edades entre 4 y 20 años. El grupo de escolares con edades entre 10 a 14 años fue el más frecuentemente derivado $(43,5 \%)$ (tabla 2).

En relación con la mejor Agudeza Visual de Lejos, en el mejor ojo con la mejor corrección óptica, 272 niños fueron analizados (tabla 3 ), considerando que otros $6(2,2 \%)$ no presentaban baja visión ya que al mejorar su corrección óptica lograban visión mayor de 0,3 . De los 272 niños con baja visión, 89 niños $(33,1 \%)$ presentaron una BV moderada, $86(31,6 \%)$ una BV severa y $67(24,6 \%)$ una BV profunda. 29 escolares $(10,7 \%)$ no se obtuvo una agudeza visual, 25 porque no logran fijan y 4 niños no cooperan por ser menores de 5 años. Considerando los 67 casos con BV profunda, $63(94,0 \%)$ pudieron ser habilitados.

En relación a los diagnósticos de ingreso, los más frecuentemente consignados como causantes de BV fueron: $41(14,7 \%)$ casos de cataratas congénitas, 30 (10,8\%) distrofias retinales, $24(8,6 \%)$ alta miopía asociado a compromiso corioretinal macular y $16(5,8 \%)$ atrofia óptica bilateral (gráfico 1). Considerando una clasificación anatómica de las patologías, se obtuvo que $61(21,9 \%)$ escolares presentan compromiso retiniano, $47(16,9 \%)$ afecciones neurooftalmológicas, 41 $(14,7 \%)$ cataratas, $34(12,2 \%)$, malformación ocular y $33(11,9 \%)$ error refractivo. Considerando los 33 escolares con error refractivo, 24 presentaban una alta miopía asociado a un daño retinal macular y 9 una alta hipermetropía con ambliopía bilateral que no corrige su visión con lentes.

Del total evaluado, 224 escolares (80,6\%) pudieron ser habilitados visualmente logrando una visión mayor de 0,4 de lejos (198 escolares) y/o de cerca (199) con ayudas de baja visión, 48 (17,3\%) no pudieron ser habilitados y $6(2,2 \%)$ mejoraron su AV basal a rango funcional con una nueva corrección óptica y no requieren ayudas de BV. En relación con los escolares habilitados por grupos etarios (gráfico 2), se constató que $90,0 \%$ de los niños de 15 años o mas pudo habilitarse y se reduce al 73,1\% en el grupo de 5 a 9 años. El grupo de menores de 5 años ( 4 casos), fue apoyado con ayudas no ópticas (atril de lectoescritura) y estimulación visual, hasta que requieran ayudas ópticas para su integración escolar.

Considerando los 224 (80,6\%) escolares que pudieron habilitarse visualmente, $124(55,4 \%)$ son hombres y $100(44,6 \%)$ mujeres. Según la visión de lejos al ingreso, $85(38,0 \%)$ presenta BV severa (tabla 3 ) y según su perimetría,122 $(54,5 \%)$ presentaron una perimetría 


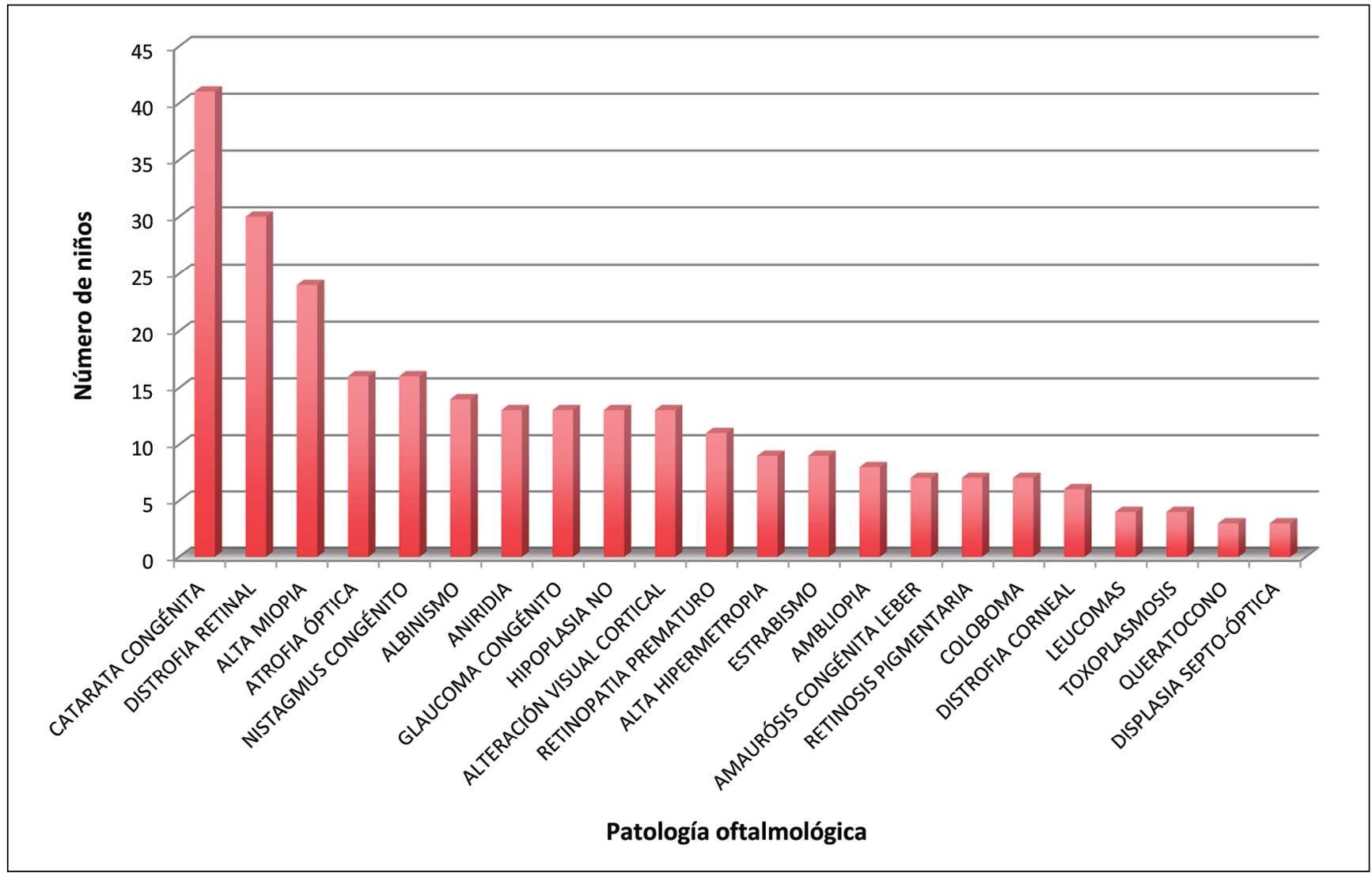

Gráfico 1. Distribución de escolares ingresados al proyecto de rehabilitación en BV de JUNAEB, de acuerdo a diagnóstico médico de ingreso.

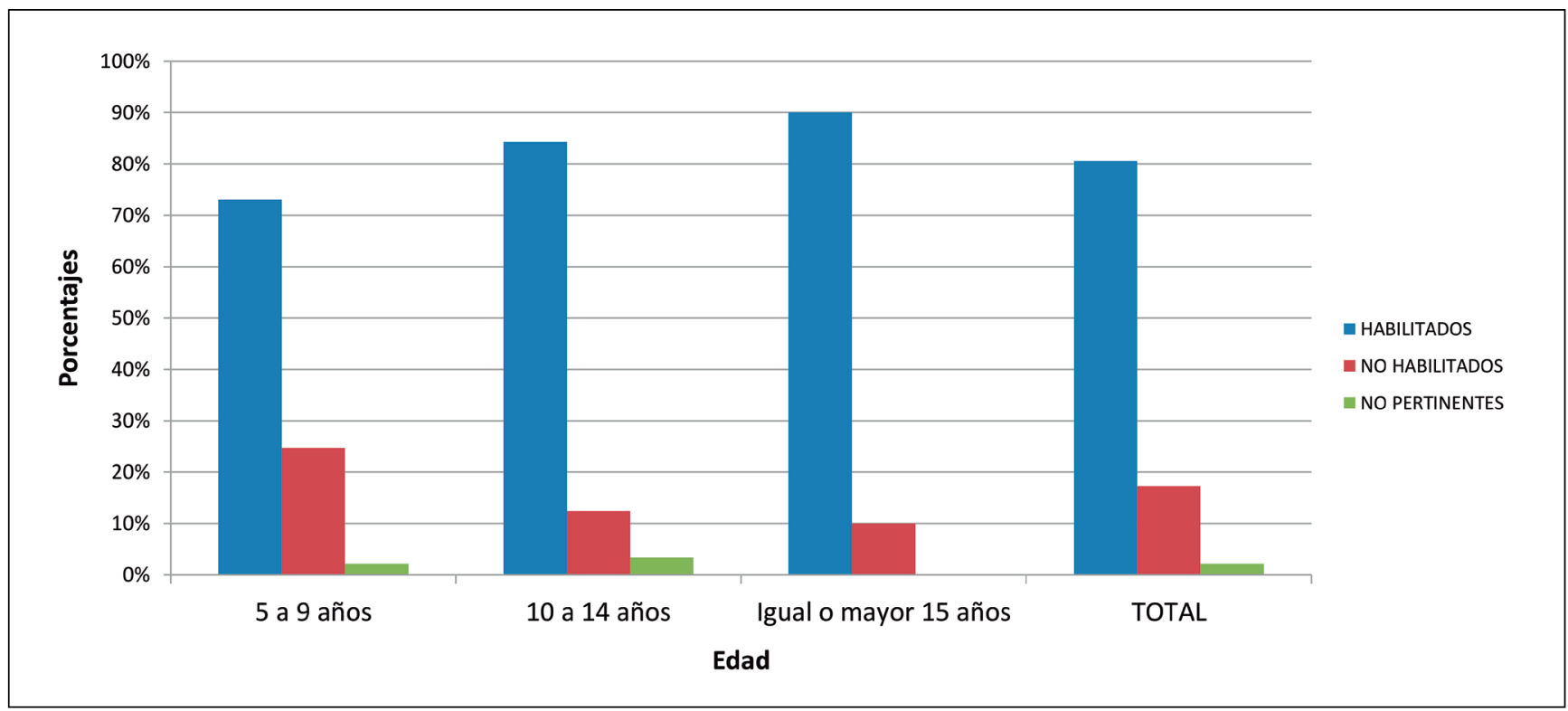

Grafico 2. Distribución de escolares ingresados al proyecto de rehabilitación en BV de JUNAEB de acuerdo a habilitación visual y grupo etario.

normal, $73(32,6 \%)$ CV tubular, $19(8,5 \%)$ defectos sectoriales del CV y 5 (2,2\%) compromiso central del CV. Sus diagnósticos de ingreso fueron: $39(17,4 \%)$ casos de catarata congénita bilateral, $26(11,6 \%)$ con distrofia retinal, $17(7,6 \%)$ con alta miopía, $16(7,1 \%)$ con nistagmus congénito y $14(5,8 \%)$ con albinismo ocular y/o oculocutáneo. Al evaluar el éxito del tratamiento de habilitación visual, 198 (88,4\%) escolares al- 
canzaron el objetivo de éxito de una AV mayor o igual a 0,4 para lejos con ayuda óptica (telescopio óptico). Otros $23(10,3 \%)$ escolares no mejoraron su visión y $3(1,3 \%)$ no requirieron ayuda óptica. Evaluando la visión de cerca, todos los escolares lograron éxito en su tratamiento logrando visión mayor de 0,4. En 199 (88.8\%) requirieron ayudas ópticas para cerca (lupas ópticas y/o electrónicas) así como no ópticas (atril de lectoescritura) y $25(11,2 \%)$ sólo ayudas no ópticas. Todos los escolares alcanzaron AV mayor o igual a 0,4 para cerca con ayudas, distribuyéndose en porcentajes similares en todas las edades, independientemente del grado de BV y perimetría.

Considerando los 48 (17,3\%) escolares que no pudieron habilitarse visualmente, 26 (54,2\%) fueron hombres y $22(45,8 \%)$ mujeres. El grupo etario entre 5 a 9 años es el más representado, con 23 (47,9\%) niños. Según la visión de lejos al ingreso, en 25 (52,1\%) casos no es posible cuantificar su AV por presentar una mala fijación asociado a daño neurológico, 14 (29,2\%) presenta BV moderada, 1 (2,1\%) BV severa y $4(8,3 \%)$ BV profunda (tabla 3 ). Los restantes $4(8,3 \%)$ niños incluidos son menores a 5 años, por lo que fueron apoyados con ayudas no ópticas (atril de lectoescritura) y estimulación visual, hasta requerir habilitación visual con ayudas ópticas para su escolarización. Los principales diagnósticos en el grupo no habilitado fueron: $10(20,8 \%)$ casos con alteración visual cortical, 5
$(10,4 \%)$ alta miopía asociado a daño corioretinal que compromete su mácula, 5 (10,4\%) glaucoma congénito, 4 (8,3\%) atrofia óptica bilateral, 4 (8,3\%) distrofia retiniana, $4(8,3 \%)$ ROP y $2(4,2 \%)$ hipoplasia del nervio óptico bilateral. En ellos no fue posible indicar ayudas ópticas para mejorar su visión. Considerando una clasificación anatómica de estos diagnósticos, el más frecuente es una condición neuroftalmológica con $18(37,5 \%)$ casos, seguida de $5(10,4 \%)$ casos de miopía con daño retinal macular, $5(10,4 \%)$ con glaucoma congénito y $5(10,4 \%)$ con malformación ocular.

Considerando los $6(2,2 \%)$ casos clasificados como no pertinentes, $3(50,0 \%)$ fueron hombres, siendo el grupo etario entre 10 a 14 años el más frecuente con $4(66,6 \%)$ niños. Sus diagnósticos de ingreso fueron: $3(50,0 \%)$ niños asociados a error refractivo por alta miopía (2) y alta hipermetropía (1), 1 (16,7\%) niños con estrabismo, $1(16,7 \%)$ con coloboma y $1(16,7 \%)$ caso asociado a catarata congénita y glaucoma. Todos mejoraron su AV con una nueva refracción y lentes ópticos, no ingresando al proyecto.

Considerando todos los diagnósticos de derivación, las patologías con mayor éxito de tratamiento fueron Aniridia, Albinismo y Nistagmus congénito, con 100\% de casos habilitados. Las patologías con menor éxito de tratamiento fueron las alteraciones visuales corticales con $23,1 \%$ habilitados, ROP con 54,5\% y Glaucoma congénito con $61,5 \%$ (gráfico 3 ).
Gráfico 3. Distribución de escolares ingresados al proyecto de rehabilitación en BV de JUNAEB de acuerdo a patología de derivación y su habilitación visual.

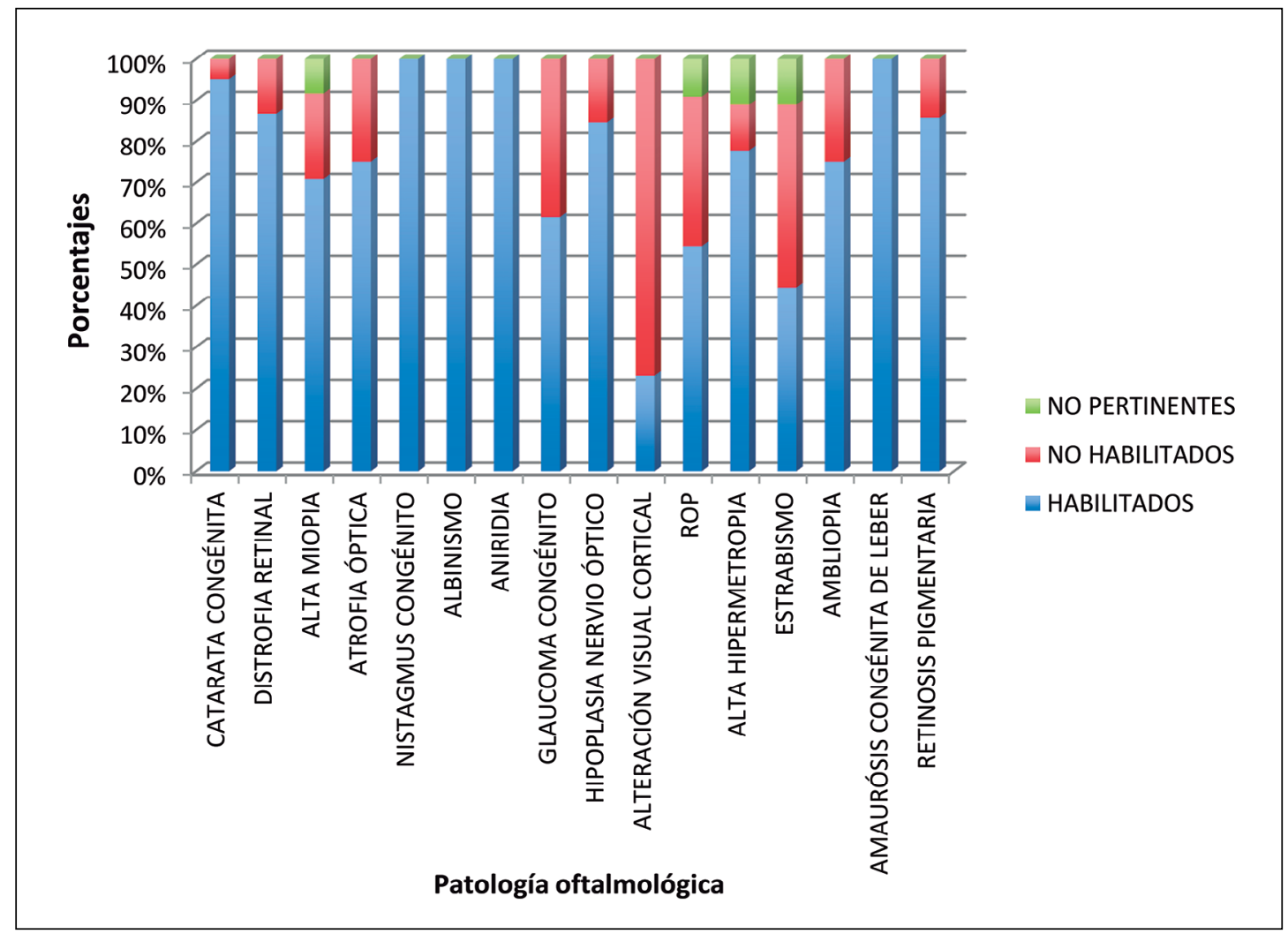




\section{Discusión}

La rehabilitación visual en niños con BV depende de las necesidades visuales, capacidades personales y remanente visual de cada niño ${ }^{10}$. Desde el punto de vista funcional, se requiere que posean un remanente visual suficiente para ver la luz y emplearla adecuadamente. Una clasificación de BV es necesaria para trabajar en su rehabilitación y en la educación de su familia y entorno. Un niño con BV profunda percibe movimientos; casos de BV severa pueden distinguir objetos medianos, sin discriminar detalles especiales o color. Un niño con BV moderada es capaz de percibir objetos pequeños o símbolos. Existe una relación inversa entre el grado de BV del niño y su independencia funcional en el colegio e interacción social ${ }^{11}$. La rehabilitación visual mejora su calidad de vida y une las ayudas de BV con el desarrollo de habilidades para su integración comunitaria. Con ayudas ópticas, no ópticas y/o electrónicas la mayoría de los niños con BV pueden acceder a la lectura y escritura en tinta ${ }^{10}$. La Organización Panamericana de Salud (OPS/OMS) destaca la importancia de la rehabilitación visual del niño, para mejorar su capacidad de lectura, asegurando su aprendizaje y educación, siendo ella de las prioridades del plan regional de salud visual ${ }^{12,13}$. En nuestro trabajo, el $80,6 \%$ de los escolares derivados fueron habilitados visualmente, considerando que lograban $\mathrm{AV}$ que les permite realizar actividades de la vida diaria en forma independiente e integrarse al sistema educacional, siendo mas importante en los escolares con BV severa, donde la habilitación fue posible en el 94\% de los casos. Según el grupos etarios, el 90,0\% de los niños mayores de 15 años se pudieron habilitar lo que se reduce al $73,1 \%$ en el grupo de 5 a 9 años, por el ingreso de niños con patologías neurológicas que no logran mejorar su AV. Solo el 2,2\% de los niños derivados no fueron pertinentes y mejoraron su visión con una nueva refracción, saliendo del grupo de baja visión pero quedan con deficiencia visual por lo cual también se les ayuda y orienta para el desarrollo de sus actividades diarias.

La prevalencia y causas de discapacidad visual infantil varía ampliamente, relacionándose con factores socioeconómicos y culturales ${ }^{11}$. Se estima que 900 niños por millón requieren tratamiento de BV, 106 intervención temprana y 230 de soporte educativo ${ }^{12}$. De 17,6 millones de niños con BV a nivel mundial, aproximadamente $60 \%$ tiene impedimentos visuales por causas tratables o prevenibles ${ }^{14}$. Describir la población atendida en un servicio de BV y sus causas a nivel local permite planificar y desarrollar estrategias dirigidas a maximizar la eficacia de los servicios y programas de rehabilitación visual disponibles e implementar estrategias preventivas para patologías prevalentes, educando a profesionales, oftalmólogos y de salud infantil.
Los avances en medicina y políticas de salud han evitado muchos casos de ceguera asociada al tracoma o deficiencia de vitamina A, pero han aumentado los casos con deficiencia visual por Catarata congénita o Glaucoma, que también son condiciones potencialmente tratables ${ }^{11}$. Existe escasa literatura sobre discapacidad visual en escolares y en su mayoría se basa en poblaciones de escuelas o centros especializados, metodologías que presentan riesgo de sesgo, especialmente en entornos vulnerables y de bajos ingresos ${ }^{15}$. En China una encuesta nacional de menores de 14 años reportó una prevalencia de baja visión de un $0,58 \%$ constatando que la catarata (48\%) y el error refractivo asociado a una ambliopía bilateral (14\%) son las principales causas $^{16,17}$. En Canadá se estimaron en 19.700(2,4\% del total) los menores de 14 años afectados con $\mathrm{BV}^{11}$, proyectándose un aumento futuro de casos asociados a condiciones congénitas o adquiridas ${ }^{2}$. Otro estudio de deficiencia visual infantil, en Nueva Zelandia, evaluó 267 niños, siendo $144(53,9 \%)$ ciegos y $123(46,1 \%)$ con BV. En ellos, las 3 principales causas de ceguera fueron una deficiencia visual cerebral $(42,4 \%)$, atrofia del nervio óptico (12,5\%) y distrofia retiniana $(9,0 \%)$, mientras que de BV fueron albinismo $(17,9 \%)$, seguido de distrofia retiniana y deficiencia visual cerebral ( $13,0 \%$ cada uno), destacando que $18,8 \%$ de los niños ciegos y $22,0 \%$ de los con BV tenían una causa evitable $^{18}$. En Nepal, de 558 niños con discapacidad visual, las causas más comunes de BV fueron el error refractivo asociado a una ambliopía (20,1\%), retinitis pigmentosa $(14,9 \%)$ y distrofia macular $(13,4 \%)$. A $86 \%$ se prescribió ayudas de BV, demostrándose que $72 \%$ de ellos mejoraban su AV para distancia de lejos y/o cerca con ayudas ${ }^{19}$.

Nuestro estudio analizó 278 niños, derivados al programa de rehabilitación en $\mathrm{BV}$ como ingreso o controles a nivel nacional durante dos años, y aunque la prevalencia de BV es baja considerando que el año 2017 de las 134.428 consultas refractivas realizadas por el programa JUNAEB, 57 niños $(0,04 \%)$ fueron derivados al programa, es importante su manejo. La primera causa de BV la catarata congénita bilateral $(14,7 \%)$, siendo estos casos una patología potencialmente tratable, por tanto, una BV secundaria prevenible. Le siguen en frecuencia las distrofias retinales $(10,8 \%)$ y alta miopía $(8,6 \%)$ asociado a daño macular, ambas condiciones no modificables. Un 3,2\% de los niños se asocia a una alta hipermetropía, siendo habilitados el 77,8\% ademas de un caso no habilitado por tener una ambliopía bilateral y otro caso que mejora su visión con corrección óptica. Otro aspecto por considerar son los casos derivados con diagnostico de ingresos poco claros como estrabismo, que produce una deficiencia unilateral o una ambliopía, pero no se describe la patología original que causa esta $\mathrm{BV}$, siendo 
imposible clasificar su real etiología. Se hace necesario establecer normas de evaluación y estandarización de los diagnósticos de derivación para atención en BV y capacitación de los Oftalmólogos prestadores.

Medir la visión de lejos y cerca es necesario para planificar la rehabilitación visual y evaluar sus resulta$\operatorname{dos}^{10}$. Cuantificar la AV en niños tiene particularidades relacionadas con su personalidad, requerimiento de aprendizaje previo de los sistemas de optotipos y la ansiedad y colaboración que prestan ante los exámenes ${ }^{20}$. $\mathrm{Su}$ rehabilitación visual debe basarse en la medición de visión de cerca, pues es fundamentalmente ésta la que requieren para trabajar en las labores escolares. No existe evidencia ni guías claras que estandaricen esta medición. Una revisión sistemática ${ }^{20}$ para determinar el impacto de una cartilla de visión cercana y los métodos para estimarla recomienda en niños hasta 3 años usar procedimientos de mirada preferencial; entre 4 a 7 años test basados en símbolos LEA y para mayores de 8 años, con respuesta verbal o que asimilen símbolos, cartillas ETDRS, porque serían más predecibles para una visión funcional ${ }^{21}$. En nuestro estudio se utilizó el test de visión próxima de Zeiss en niños letrados y se consideró éxito lograr AV mayor o igual a 0,4 con ayuda óptica, por ser una recomendación de la Fundación ONCE, reconocida institución española dedicada a la rehabilitación visual ${ }^{10} \mathrm{y}$ porque esta visión les permite leer e ingresar a la educación regular. Los alumnos evaluados y habilitados en programa de baja visión logran realizar sus actividades educativas como leer sus libros escolares y copiar de la pizarra con las ayudas ópticas y no ópticas entregadas. Con esto pueden acceder a la lectoescritura en tinta y continuar así su escolaridad considerando que sin estas ayudas se deben manejar en sistema braille o macrotipo.

El beneficio del uso de ayudas ópticas de lectura para niños y adolescentes con BV esta consolidado en recomendaciones de expertos y equipos de trabajo en $\mathrm{BV}$, pese a ello, aún no existe evidencia de calidad para recomendar ayudas específicas, principalmente por falta de estudios randomizado. Los futuros estudios debieran uniformar sus resultados para facilitar comparaciones en los metaanálisis. Los resultados funcionales de los tratamientos de BV no son fáciles de unificar ${ }^{22}$ por la presencia de variables no estandarizadas, como visión, facilidad de lectura primaria (velocidad de lectura y comprensión) y duración, aprendizaje, tamaño de impresión, así como cuestionarios o análisis de costo efectividad entre otros. Para medir el éxito del tratamiento un estudio ${ }^{23}$ evaluó cambios en la visión funcional de 183 niños, usando cuestionario PRASAD II, al ingreso y al 3-4 mes posterior, demostrando que un servicio de BV mejora la función visual independiente de su causa y enfatiza la necesidad de rehabilitar visualmente a niños con BV. Nuestra percepción es que estos niños se han integrado al sistema educacional con ayudas de BV, entregadas sin costo por JUNAEB, logrando una mayor equidad pero se requieren a futuro incluir cuestionarios en el seguimiento del proyecto de JUNAEB para evaluar la mantención del éxito alcanzado, después de la adaptación del niño a su ayuda visual y el impacto que ésta representa en su integración educacional y comunitaria. Se plantea también identificar causas de falla de la rehabilitación y no uso de ayudas con el fin de planificar estrategias para prevenirlas.

Para desarrollar políticas publicas falta determinar la efectividad de los tratamientos en niños, pues la literatura disponible esta dirigida mayormente a adultos $^{24}$. Una revisión sistemática buscó objetivar el beneficio de la rehabilitación visual en niños, obteniendo sólo 28 artículos que cumplían criterios de inclusión. Mostró que habilitar visualmente a niños con BV es muy importante, pero existe poca literatura al respecto, generalmente reportes de casos. Otra revisión ${ }^{22}$ no permitió desarrollar recomendaciones por falta de estudios randomizados, pero destacó la importancia de la rehabilitación visual infantil.

Existen algunas limitaciones del estudio como es contar con una muestra pequeña y que son referidas por lo cual no es posible estimar prevalencias ni conocer la carga de las diferentes patologías en la baja visión, pues esta asociado a la pesquisa espontanea y al conocimiento del programa de parte del medico tratante. Por otro lado, consideramos el diagnostico de derivación del medico tratante, como el diagnostico principal para los análisis, en parte por ser una muestra derivada del programa JUNAEB presente en todo Chile. También lo anterior genera que los diagnosticos no estén estandarizados y algunas causas no están claras como por ejemplo ambliopía sin causa o estrabismo que puede ser secundario o un diagnostico mal definidos como alteración visual cortical, y para corregir en parte esto se realizó una clasificación anatómica.

En conclusión, se requiere implementar servicios de rehabilitación de BV para ayudar a los niños a ser independientes, evitando pérdida de oportunidades educacionales, laborales y deterioro de calidad de vida. Desde el año 1992, JUNAEB realiza detección y manejo de deficiencias visuales asociadas a error refractivo $^{25} \mathrm{y}$, desde el año 2015, incorpora el proyecto de rehabilitación visual, habilitando a escolares con BV mediante entrega ayudas ópticas, no ópticas y electrónicas, sin costo para beneficiarios. El 80,6\% de los niños tuvieron éxito en su rehabilitación visual lo cual demuestra la eficiencia de este programa con la habilitación del niño con ayudas ópticas, lo que le permite ver de cerca. Nuestro desafío es mantener este modelo de atención de BV infantil de cobertura nacional, involucrarlo con otros proyectos de integración, trabajar y capacitar a profesores y apoderados y edu- 
car al oftalmólogo prestador para derivar adecuada y precozmente a los afectados. Todo ello debe generar redes que permitan optimizar los recursos estatales dirigidos a estos escolares.

\section{Responsabilidades Éticas}

Protección de personas y animales: Los autores declaran que los procedimientos seguidos se conformaron a las normas éticas del comité de experimentación humana responsable y de acuerdo con la Asociación Médica Mundial y la Declaración de Helsinki.
Confidencialidad de los datos: Los autores declaran que han seguido los protocolos de su centro de trabajo sobre la publicación de datos de pacientes.

\section{Derecho a la privacidad y consentimiento informa- do: Los autores han obtenido el consentimiento in- formado de los pacientes y/o sujetos referidos en el artículo. Este documento obra en poder del autor de correspondencia.}

\section{Conflicto de intereses}

Los autores declaran no tener conflicto de intereses.

\section{Referencias}

1. Organización Mundial de la Salud (Internet). OMS; c2017. Ceguera y Discapacidad Visual. Disponible en: http://www.who.int/mediacentre/ factsheets/fs282/es/

2. Brilliant, R. Essentials of low vision practice. Boston: ButterworthHeinemann. 1999.

3. Barría F, Silva JC, Limburg H, Muñoz D. Análisis de la prevalencia de ceguera y sus causas determinados mediante encuesta rápida de ceguera evitable (RAAB) VIII región, Chile. Arch. Chil. Oftalmol. 2008;64(1-2):69-78.

4. Gogate P, Bhusan S, Ray S, Shinde A. Impact of correcting visual impairment and low vision in deaf-mute students in Pune, India. Indian J Ophthalmol. 2016;64:898-903.

5. Inde K, Backman O. El adiestramiento de la visión subnormal. Madrid: ONCE España. 1988.

6. Barria F, Conte F, Muñoz S, Leasher JL, Silva JC. Prevalence of refractive error and spectacle coverage in schoolchildren in two urban areas of Chile. Rev Panam Salud Publica. 2018;42:e61. https://doi. org/10.26633/RPSP.2018.61.

7. Maul E, Barroso S, Muñoz SR, Sperduto $\mathrm{RD}$, Ellwein LB. Refractive error study in children: results from La Florida, Chile. Am J Ophthalmol. 2000;129(4):445-54.

8. International Statistical Classification of Diseases and Related Health Problems 10th Revision (ICD-10)-WHO Version for ;2016. Disponible en: http://apps.who. int/classifications/icd10/browse/2016/en\#/ H54.

9. Faye E. Clínica de la Baja Visión. 2da ed 1984(traducción al español ONCE 1987). $15 \mathrm{p}$.
10. ONCE. Apuntes sobre Rehabilitación Visual: Protocolo. 1ra ed. Madrid España; $1994.175 \mathrm{p}$.

11. Markowitz SN. State-of-the-art: low vision rehabilitation. Can J Ophthalmol. 2016;51:59-66

12. Organización Panamericana de la Salud, Organización Mundial de la Salud (Internet). Washington DC: OPS OMS; c2017. Prevencion de la ceguera y salud ocular. Disponible en: http://www.paho.org/hq/index. php?option $=$ com_content\&view $=$ article \&id $=244 \% 3$ A2008-prevention-blindnesseye-care\&catid $=1177 \% 3$ Apreventionblindness-eye\&Itemid $=39604 \&$ lang $=$ es\&li mitstart $=0$.

13. Organización Panamericana de la Salud, Organización Mundial de la Salud (Internet). Washington DC: OPS OMS; c2017. Plan de acción para la prevención de la ceguera y de las deficiencias visuales. Disponible en: www.paho.org/hq/index. php?option $=$ com_docman\&task $=$ doc... gid... www.v2020la.org/.../index.php?... plan-de-accion-para-la-prevencion-de-laceguera.

14. Pascolini D, Mariotti S. Global estimates of visual impairment. Br J Ophthalmol. 2011;1:1

15. Solebo AL, Teoh L, Rahi J. Epidemiology of Blindness in Children. Arch Dis Child. 2017;0:1-5. DOI: 10.1136/ archdischild-2016-310532

16. Gao G, Yu M, Dai J, et al. Demographic and clinical characteristics of a paediatric low vision population in a low vision clinic in China. Clin Exp Optom. 2016;99:274-9. DOI:10.1111/cxo.12294.

17. Zhang SY, Zou LH, Gao YQ, Di Y, Wang $\mathrm{XD}$. National epidemiological survey of blindness and low vision in China. Chin Med J (Engl) 1992;105:603-8.
18. Chong CF, McGhee CN, Dai S. A Cross-sectional Study of Prevalence and Etiology of Childhood Visual Impairment in Auckland, New Zealand. Asia Pac J Ophthalmol. 2014;3: 337-42.

19. Uprety S, Khanal S, Morjaria P, Puri L. Profile of paediatric low visión: a retrospective study from Nepal. Clin Exp Optom. 2016; 99: 61-5.

20. Huurneman B, Boonstra FN. Assessment of near visual acuity in 0-13 year olds with normal and low vision: a systematic review. BMC Ophthalmology. 2016;16:215. DOI: 10.1186/s12886-0160386-y.

21. Brandão AO, Andrade GM, Vasconcelos GC, Rossi LD, Saliba GR. Instruments for evaluation of functionality in children with low vision: a literature review. Arq Bras Oftalmol. 2017;80(1):59-63.

22. Barker L, Thomas R, Rubin G, Dahlmann-Noor A. Optical reading aids for children and young people with low vision. Cochrane Database of Systematic Reviews. 2015;3. DOI: 10.1002/14651858. CD010987.pub2.

23. Gothwal VK, Sumalini R, Bharani S. Assessing the effectiveness of low vision rehabilitation in children: an observational study. Invest Ophthalmol Vis Sci. 2015;56:3355-60. DOI: 10.1167/ iovs.14-15760.

24. Chavda S, Hodge W, Si F, Diab K. Lowvision rehabilitation methods in children: a systematic review. Can J Ophthalmol. 2014;49:71-3.

25. Programa de salud escolar de la Junta Nacional de Auxilio Escolar y Becas. (Internet). Santiago: JUNAEB; 2017. Disponible en: http://www.junaeb.cl/ prontus_junaeb/site/edic/base/port/salud. html. 